05,01

\title{
Вклады магнитных фаз в диаграмму FORC в магнитах (NdDy)(FeCo)B
}

\author{
(C) В.В. Кучеряев ${ }^{1}$, Е.И. Куницына ${ }^{2}$, Р.А. Валеев ${ }^{1}$, Д.В. Королев ${ }^{1}$, В.П. Пискорский ${ }^{1}$, Р.Б. Моргунов ${ }^{1,2, \uparrow}$ \\ ${ }^{1}$ Всероссийский институт авиационных материалов, \\ Москва, Россия \\ ${ }^{2}$ Институт проблем химической физики РАН, \\ Черноголовка, Россия \\ ฯE-mail: morgunov2005@yandex.ru
}

(Поступила в Редакцию 10 мая 2018 г.)

Обсуждается взаимосвязь микромагнитной структуры, вкладов различных кристаллических фаз и гистеронов, полученных методом кривых обратного намагничивания первого порядка (First Order Reversal Curves) в спеченных магнитах $(\mathrm{NdDy})(\mathrm{FeCo}) \mathrm{B}$. Построена карта распределения параметров частичных петель магнитных гистерезисов, позволяющих разделить вклады различных спиновых ансамблей в намагниченность.

ФГУП „ВИАМ““. Работа выполнена в рамках реализации комплексного научного направления 11.1 „Термостабильные магнитотвердые материалы и математические модели расчета их температурных характеристик для навигационных приборов нового поколения“ („Стратегические направления развития материалов и технологий их переработки на период до 2030 г.“).

DOI: $10.21883 /$ FTT.2018.12.47347.127

\section{1. Введение}

Сверхсильные магниты семейства $R E-T M-B(R E-$ редкоземельный металл, $T M$ - переходный металл) широко известны самым высоким энергетическим произведением $(B H)_{\max }$, где $B-$ индукция магнитного поля, пропорциональная остаточной намагниченности $M_{R E M}$, a $H$ - напряженность магнитного поля, пропорциональная коэрцитивной силе $H_{C}$. Техническая характеристика магнитов, как правило, исчерпывается упомянутыми выше величинами. Новые физические методы исследования магнитов приносят более разнообразные параметры, позволяющие больше судить о внутренних взаимодействиях в магнитной системе, состоящей из ансамблей намагниченных частиц (зерен) и разделенных на области намагничивания (домены и фаза обратной намагниченности). Распределение и взаимодействие этих объектов многообразно и сложно. Именно оно определяет конечные свойства постоянных магнитов, которые нуждаются в совершенствовании.

Одним из методов изучения межчастичных взаимодействий и коллективов частиц в магните является широко распространенный метод кривых перемагничивания первого порядка, более известный как FORC (First Order Reversal Curves) [1-5]. Метод впервые был предложен в теоретической работе [1] и основан на анализе кривых перемагничивания, получаемых при уменьшении магнитного поля до значения $H_{b}$, выводящем систему из насыщения (рис. 1). При этом получение двумерного распределения намагниченности $M\left(H_{b} ; H_{a}\right)$ как функции поля $H_{b}$ и текущего поля $H_{a}$ на кривой размагничивания позволяет успешно разделять вклады различных подсистем в магнитные свойства образца даже без глубокого анализа [1-5]. Однако кроме феноменологического подхода в литературе развиты изящные математические методы извлечения физической информации из петель гистерезиса, причем довольно универсальные и пригодные не только в магнетизме [1-3]. Применение FORC для анализа магнитных свойств постоянных магнитов системы $R E-T M-B$ показало продуктивность этого подхода $[4,5]$. Физические основания для возможности разделения вкладов магнитных подсистем в спеченных магнитах заключаются в том, что в классическом магните $\mathrm{Nd}_{2} \mathrm{Fe}_{14} \mathrm{~B}$ магнитная корреляционная длина составляет $d=(A / K)^{1 / 2}=1.35 \mathrm{~nm}$, что сопоставимо с межзеренным расстоянием и создает особенности намагничивания вблизи краев зерен $(A-$ константа обменного взаимодействия, $K$ - константа магнитокристаллической анизотропии). Кроме того, в постоянных магнитах $R E-T M-B$ в зависимости от системы легирования помимо основной фазы 2-14-1 содержатся магнитные фазы, которые могут давать соответствующие различимые отклики на диаграмме FORC.

Цель работы заключается в экспериментальном получении серий кривых перемагничивания и их анализе

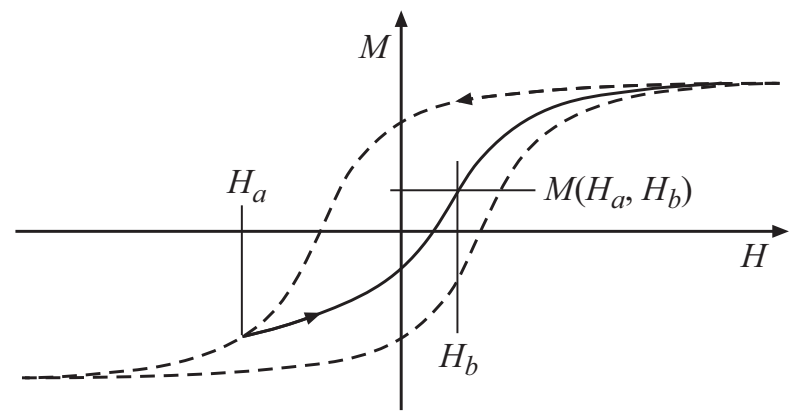

Рис. 1. Схематическое изображение петли гистерезиса и параметров $H_{b}$ и $H_{a}$ метода FORC (пояснения см. в тексте). 
для разделения вкладов магнитных подсистем кристалла (различных фаз, зерен, зародышей фазы обратной намагниченности и др.) в намагниченность спеченных магнитов семейства $(\mathrm{NdDy})(\mathrm{FeCo}) \mathrm{B}$.

\section{2. Методика}

В качестве объекта исследования были выбраны спеченные магниты $\left(\mathrm{Nd}_{0.7} \mathrm{Dy}_{0.3}\right)_{16}\left(\mathrm{Fe}_{0.8} \mathrm{Co}_{0.2}\right)_{78} \mathrm{~B}_{6}$. На них были проведены нами ранее систематические исследования магнитных свойств, а их основные магнитные характеристики хорошо известны [6-10]. Образцы получали путем спекания предварительно прессованной заготовки из порошка с размером частиц $\sim 10-50 \mu \mathrm{m}$ в высокотемпературной вакуумной печи. Размер зерен основной магнитной фазы находился в интервале от 10 до $20 \mu \mathrm{m}$. Подробная методика получения исследуемых магнитов описана в работах $[9,10]$. Метод FORC, используемый в данной работе, позволяет проводить разделение вкладов в намагниченность образцов от различных структурно-фазовых составляющих, если есть данные по коэрцитивной силе и намагниченности насыщения, полученных из петли гистерезиса этих добавок. В работе [11] подобным образом проведено разделение вкладов в намагниченность керамического соединения $\mathrm{BaFe}_{2} \mathrm{O}_{4}$, феррита $\mathrm{Sr}$ и $\mathcal{\gamma}-\mathrm{Fe}_{2} \mathrm{O}_{3}$.

Все измерения магнитного момента образцов проводили с помощью магнитометра MPPMS 5XL Quantum design при температуре $T=300 \mathrm{~K}$. Внешнее магнитное поле было направлено вдоль легкой оси намагничивания образца. Исследование локального состава фаз проводили методом качественного и количественного микрорентгеноспектрального анализа (MPCA) на сканирующем электронном микроскопе „SUPERPROB-733“ („JSMA-733“, фирма JEOL, Япония) с применением энергодисперсионного анализатора INCAENERGY (Англия). Локальность анализа - $1 \mu \mathrm{m}^{2}$, глубина анализа $1 \mu \mathrm{m}$. Количественный фазовый анализ проводили на порошковых не текстурованных образцах с использованием дифрактометра ДРОН-3М, излучением $\mathrm{Cu} K_{\alpha}$.

\section{3. Экспериментальные результаты и обсуждение}

Построение диаграммы FORC проводилось в следующем порядке:

- намагничивание образца в поле насыщения $H_{\text {sat }}=50 \mathrm{kOе}$ и последующее уменьшение напряженности внешнего магнитного поля до значения $H_{a}$, отличающегося для каждой следующей кривой намагничивания;

- развертка магнитного поля от разных значений $H_{a}$ до $H_{\text {sat }}$ с постоянной скоростью и запись магнитного момента. Шаг изменения $H_{a}$ был постоянен и составлял $1.5 \mathrm{kOe}\left(H_{a}\right.$ - стартовое поле, а $H_{b}$ - текущее поле для данной кривой FORC, изменяемое с шагом $1.5 \mathrm{kOe})$.

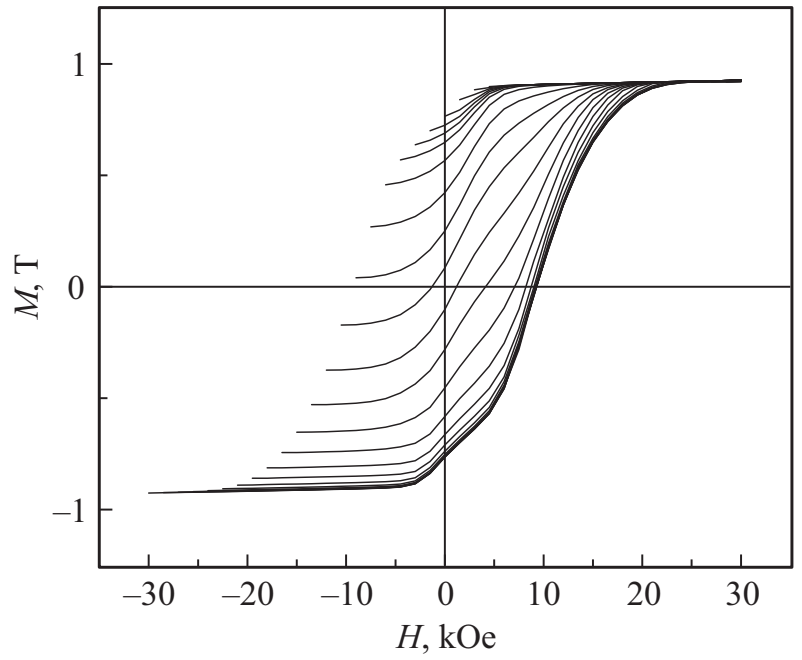

Рис. 2. Частичные петли гистерезиса образца $\left(\mathrm{Nd}_{0.7} \mathrm{Dy}_{0.3}\right)_{16}\left(\mathrm{Fe}_{0.8} \mathrm{Co}_{0.2}\right)_{78} \mathrm{~B}_{6}$, полученные при $300 \mathrm{~K}$

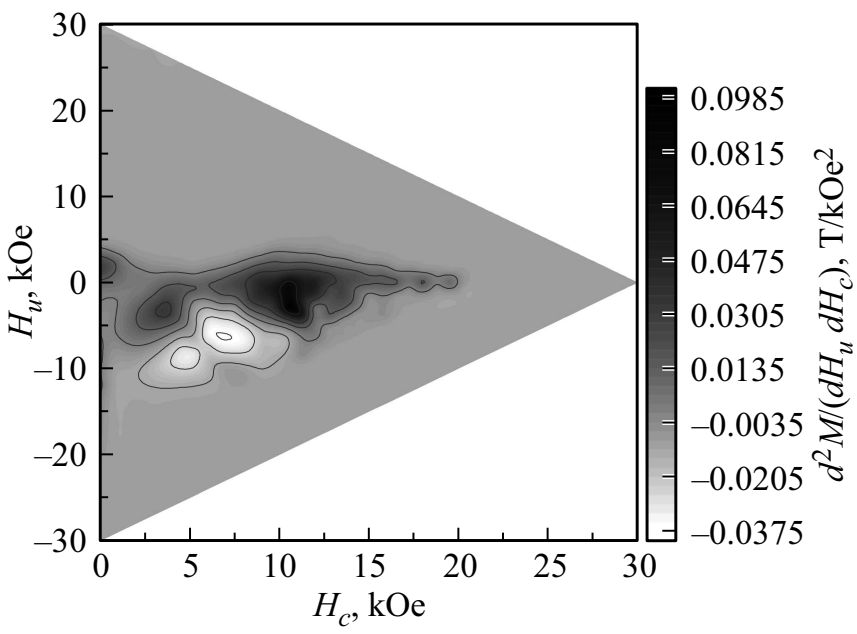

Рис. 3. Поле распределения $\rho\left(H_{c} ; H_{u}\right)$ образца $\left(\mathrm{Nd}_{0.7} \mathrm{Dy}_{0.3}\right)_{16}\left(\mathrm{Fe}_{0.8} \mathrm{Co}_{0.2}\right)_{78} \mathrm{~B}_{6}$ при $300 \mathrm{~K}$.

Диапазон значений $H_{a}$ лежал в интервале от $-30 \mathrm{kOe}$ до $30 \mathrm{kOe}$ (рис. 2);

- формирование массива данных в матрицу, выражающую собой функцию двух переменных $M\left(H_{a} ; H_{b}\right)$;

- смена координат $H_{a}, H_{b}$ на $H_{c}=\left(H_{b}-H_{a}\right) / 2$ и $H_{u}=\left(H_{b}+H_{a}\right) / 2$.

Распределение FORC представляет собой смешанную вторую производную намагниченности по стартовому и текущему полям

$$
\rho\left(H_{u}, H_{c}\right)=\frac{\partial^{2} M\left(H_{u}, H_{c}\right)}{\partial H_{u} \partial H_{c}},
$$

где $H_{u}$ - характеризует распределение полей взаимодействия спиновых ансамблей, а $H_{c}-$ соответствует распределению полей переключения намагниченностей локальных областей. 


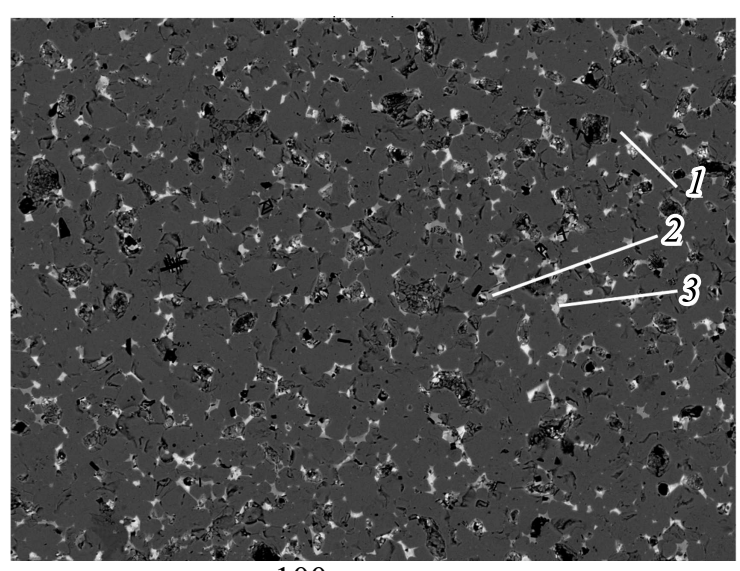

$100 \mu \mathrm{m}$

Рис. 4. Микроструктура спеченого магнита $\left(\mathrm{Nd}_{0.7} \mathrm{Dy}_{0.3}\right)_{16}\left(\mathrm{Fe}_{0.8} \mathrm{Co}_{0.2}\right)_{78} \mathrm{~B}_{6}$.

На рис. 3 представлено распределение параметра $\rho\left(H_{u} ; H_{c}\right)$ исследуемого магнита в координатах „поле взаимедоействия-поле переключения“. Как видно из рис. 3 распределение FORC исследуемого магнита представлено полем, обладающим четырьмя экстремумами, двумя положительными и двумя отрицательными.

Рассмотрим сначала отрицательные максимумы $\rho\left(H_{u} ; H_{c}\right)$. Отрицательные значения, очевидно, характеризуют такие ансамбли спинов, которые уменьшают намагниченность с ростом поля и могут отвечать, например, такой ситуации, когда намагниченность падает за время ее измерения в результате магнитной релаксации, как это было упомянуто в [3].

Рассмотрим теперь положительные максимумы $\rho\left(H_{u} ; H_{c}\right)$. В окрестностях нулевых значений $H_{c}$ присутствует область значений $\rho\left(H_{u} ; H_{c}\right)$, отличных от нуля. Данная область соответствует обратимой намагниченности, пик которой приходится на значения $H_{u}=2 \mathrm{kOе}$. Согласно [3], значения $\rho$, отличные от нуля
Химический состав и объемная доля фаз, содержащихся в исследуемом магните $\left(\mathrm{Nd}_{0.7} \mathrm{Dy}_{0.3}\right)_{16}\left(\mathrm{Fe}_{0.8} \mathrm{Co}_{0.2}\right)_{78} \mathrm{~B}_{6}$, определенные методом количественного микрорентгеноспектрального анализа на аппарате „SUPERPROB-733“ с применением энергодисперсионного анализатора INCAENERGY

\begin{tabular}{c|c|r|r|c|c|c}
\hline \multirow{2}{*}{ Фаза } & \multirow{2}{*}{$\begin{array}{c}\text { Объемная } \\
\text { доля,\% }\end{array}$} & \multicolumn{4}{|c|}{ Содержание элементов, } & \multirow{2}{*}{ mass\% mass\% } \\
\cline { 3 - 6 } & & $\mathrm{Fe}$ & \multicolumn{1}{|c|}{$\mathrm{Co}$} & $\mathrm{Nd}$ & $\mathrm{Dy}$ & \\
\hline 1 & 88.5 & 61.4 & 9.9 & 22.7 & 6.0 & 100.0 \\
2 & 4.7 & 48.4 & 12.8 & 31.7 & 5.8 & $98.7^{*}$ \\
3 & 3.4 & 7.0 & 1.1 & 66.7 & 9.5 & $84.3^{*}$
\end{tabular}

Примечание. * - остальное от $100.0 \%$ - кислород.

вблизи нулевых значений поля переключения $H_{c}$, также свидетельствуют о влиянии размагничивающего фактора. Подробное исследование влияния формы образца на поле распределения FORC в магнитах с основной магнитной фазой $\mathrm{Nd}_{2} \mathrm{Fe}_{14} \mathrm{~B}$ проведено в работе [5], в которой показано, что изменение формы образца с пластины на кубическую форму, позволяет исключить из распределения псевдогистероны, вызванные размагничивающим фактором. Поэтому для построения кривых перемагничивания первого порядка был использован образец с размагничивающим фактором $N=0.33$.

Так же на рис. 3 наблюдаются два положительных экстремума значений $\rho\left(H_{u} ; H_{c}\right)$ с координатами $H_{c}, H_{u}$ : $10 \mathrm{kOe},-3 \mathrm{kOe}$ и $3 \mathrm{kOe},-3.5 \mathrm{kOe}$ соответственно. Наличие подобных пиков свидетельствует о присутствии двух магнитных фаз, дающих вклад в петлю гистерезиса исследуемого магнита, причем коэрцитивная сила данных фаз составляет около $10 \mathrm{kOе}$ и $3.5 \mathrm{kOе}$ соответственно.

Для определения фазового состава исследуемого магнита был проведен микрорентгеноспектральный анализ, результаты которого представлены на рис. 4. Обнаружены три фазы, химический состав и объемные доли которых представлены в таблице. По химическому составу

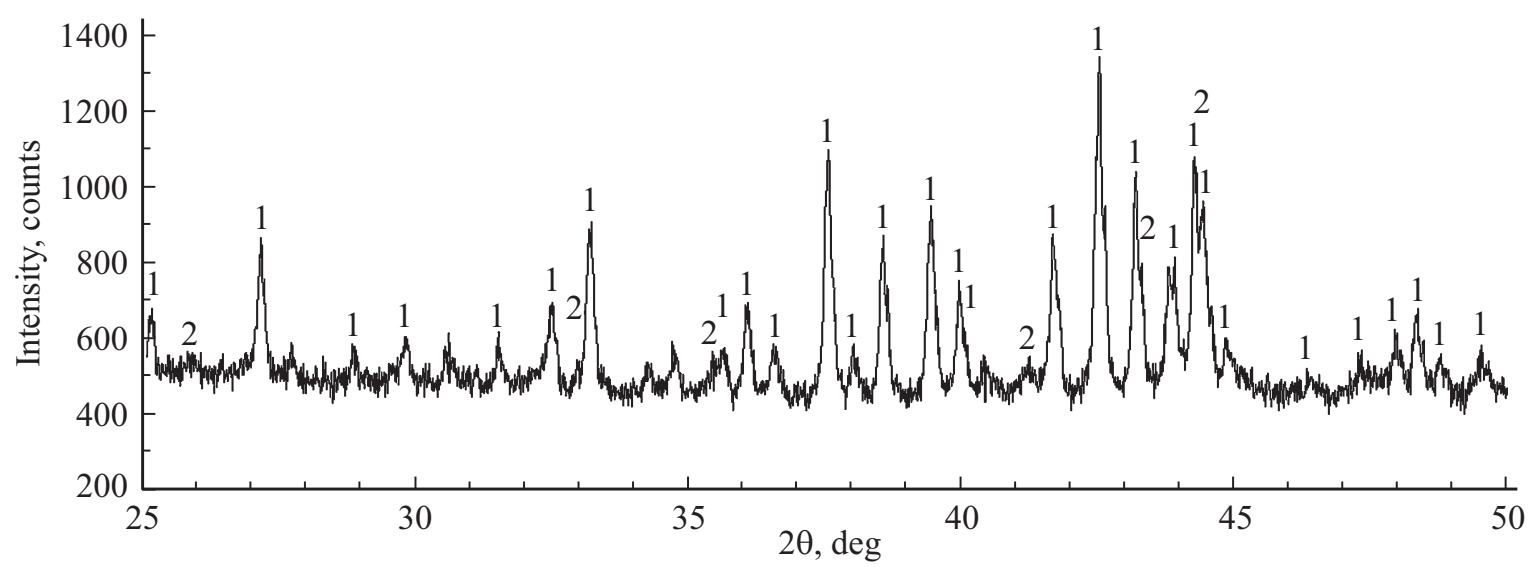

Рис. 5. Результаты рентгенофазового анализа образца $\left(\mathrm{Nd}_{0.7} \mathrm{Dy}_{0.3}\right)_{16}\left(\mathrm{Fe}_{0.8} \mathrm{Co}_{0.2}\right)_{78} \mathrm{~B}_{6}$, выполненые с использованием дифрактометра ДРОН-3М, излучением $\mathrm{Cu} K_{\alpha}$, цифрами обозначены пики соответствующие фазам, присутствующих в исследуемом образце: 1) $2-14-1,2) 1-4-1$. 
фаза 1 соответствует основной магнитной фазе 2-14-1, объемная доля которой составляет $88.5 \%$. Фаза 2 по химическому составу близка к соединениям гомологического ряда $R E_{n+1} T M_{3 n+5} B_{2 n}$, объемная доля $-4.7 \%$. Также в образце присутствует фаза 3 , объемная доля $-3.4 \%$, обогащенная РЗМ с содержанием кислорода до $15 \%$. Объемная доля пор, возникших при механической обработке поверхности образца, составляет $3.4 \%$. Для определения типа фазы 2, обнаруженной в исследуемом магните, был проведен рентгенофазовый анализ, результаты которого представлены на рис. 5. Согласно данным анализа в сплаве $\left(\mathrm{Nd}_{0.7} \mathrm{Dy}_{0.3}\right)_{16}\left(\mathrm{Fe}_{0.8} \mathrm{Co}_{0.2}\right)_{78} \mathrm{~B}_{6}$ присутствует фаза 1-4-1.

Согласно данным работы [12] коэрцитивная сила $H_{c}$ связана со средним размером зерен $D$ в магнитах $\mathrm{NdFeB}$ следующим соотношением:

$$
H_{c}=\frac{\gamma}{M_{s}} \frac{1}{D},
$$

где $\gamma$ - энергия доменной стенки, которая была определена стереологическим методом [13], по изображению, полученным методом магнитосиловой микроскопии, и равна $\sim 28 \mathrm{erg} / \mathrm{cm}^{2}, 4 \pi M_{s}-$ намагниченность насыщения, равная $8 \mathrm{kG}, D-$ средний размер зерна основной магнитной фазы. Согласно анализу рис. 4, средний размер зерна основной магнитной фазы составляет $\sim 10 \mu \mathrm{m}$. Из формулы (2) следует, что расчетное значение коэрцитивной силы исследуемого магнита при таких размерах зерен составляет $\sim 44 \mathrm{kOe}$.

На рис. 6 представлена гистограмма распределения зерен основной магнитной фазы в объеме исследуемого образца, при статистической обработке распределения использовались линейные размеры 300 зерен. С использованием выражения (2) и поправочного коэффициента, рассчитанного из сравнения эмпирического и расчетного значения коэрцитивной силы, построена гистограмма распределения $H_{c}$ от размера зерна основной магнитной фазы, рис. 7, $a$. Из распределения на рис. 7, $a$ видно, что максимальное и минимальное значения коэрцитивной

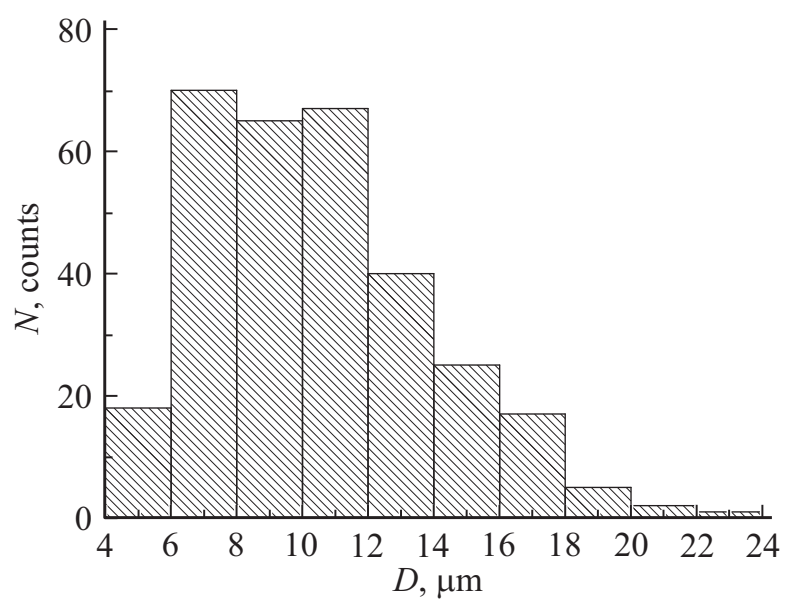

Рис. 6. Распределение зерен по диаметру в образце $\left(\mathrm{Nd}_{0.7} \mathrm{Dy}_{0.3}\right)_{16}\left(\mathrm{Fe}_{0.8} \mathrm{Co}_{0.2}\right)_{78} \mathrm{~B}_{6}$.
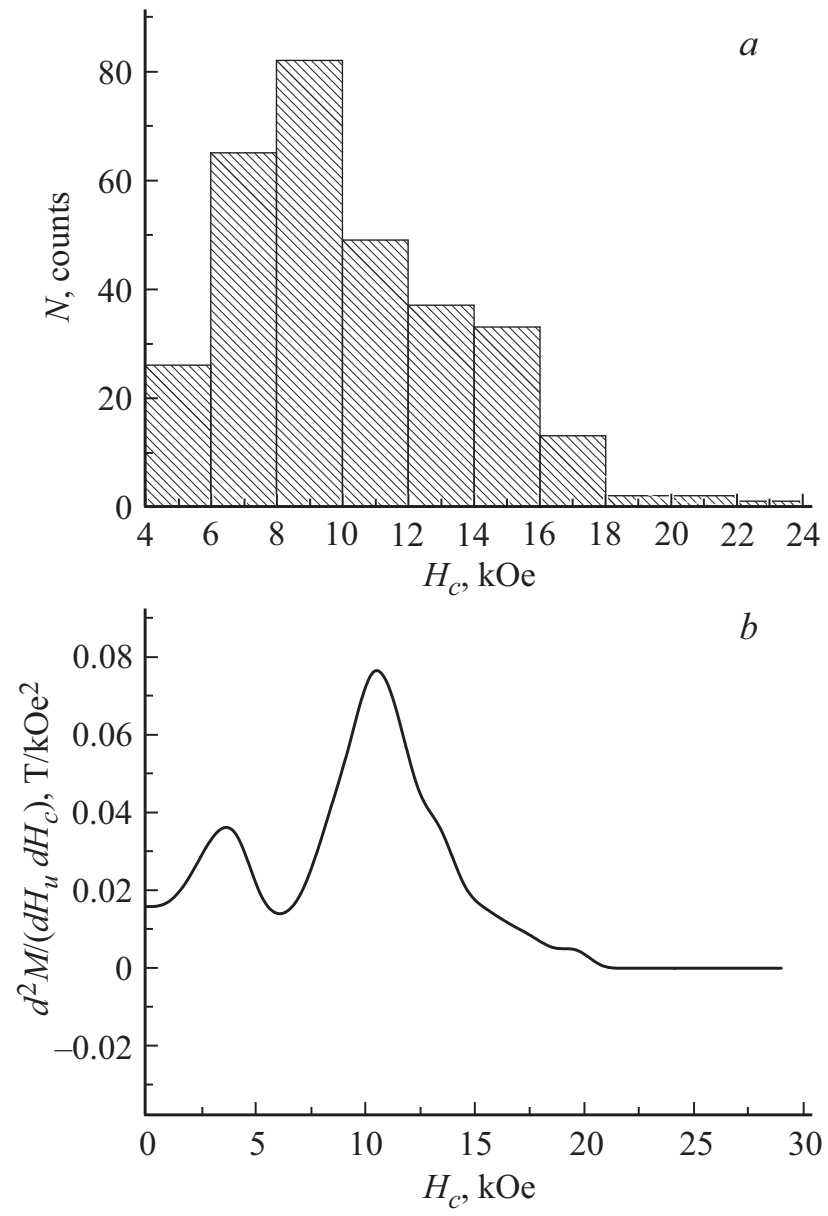

Рис. 7. Распределение коэрцитивной силы в образце $\left(\mathrm{Nd}_{0.7} \mathrm{Dy}_{0.3}\right)_{16}\left(\mathrm{Fe}_{0.8} \mathrm{Co}_{0.2}\right)_{78} \mathrm{~B}_{6}$ в зависимости от диаметра зерна основной магнитной фазы $(a)$, двухмерное распределение поле переключения $H_{c}$ на диаграмме FORC с $H_{u}=-2,0 \mathrm{kOe}(b)$.

силы зерен составляют $24 \mathrm{kOe}$ и $4 \mathrm{kOe}$ соответственно. Полученные значения совпадают с границами распределения значений поля переключения на диаграмме FORC (рис. 7, b). Данное совпадение позволяет считать, что гистерон с центром при $H_{c}=10 \mathrm{kOe}$ характеризует фазу 2-14-1, а гистерон с центром при $H_{c}=3 \mathrm{kOe}$ характеризует вклад фазы 1-4-1.

\section{4. Выводы}

На диаграмме обратной намагниченности FORC обнаружены максимумы, отвечающие вкладам двух фаз 1-4-1 и 2-14-1. Наблюдаются также максимумы, отвечающие отрицательным значениям второй производной магнитного момента, вызванные магнитной релаксацией в процессе измерений магнитного момента. Расчет распределений по размерам зерен, показывает, что ширины гистеронов (максимумов на диаграммах FORC) обусловлены распределением критических полей переключения намагниченности зерен. 


\section{Список литературы}

[1] I.D. Mayergoyz. Phys. Rev. Lett. 56, 1518 (1986).

[2] C.R. Pike, A.P. Roberts, K.L. Verosub. J. Appl. Phys. 85, 6660 (1999).

[3] C.R. Pike, C.A. Ross, R.T. Scalettar, G. Zimanyi. Phis. Rev. B 71, 134407 (2005).

[4] M. Ito, M. Yano, N. Sakuma, H. Kishimoto, A. Manabe, T. Shoji, A. Kato, N.M. Dempsey, D. Givord, G.T. Zimanyi. AIP Adv. 6, 056029 (2016).

[5] T. Schrefl, T. Shoji, M. Winklhofer, H. Oezelt, M. Yano, G. Zimanyi. J. Appl. Phys. 111, 07A728 (2012).

[6] E.N. Kablov, O.G. Ospennikova, D.E. Kablov, V.P. Piskorskii, E.I. Kunitsyna, A.I. Dmitriev, R.A. Valeev, D.V. Korolev, I.I. Rezchikova, A.D. Talantsev, R.B. Morgunov. J. Appl. Phys. 117, 243903 (2015).

[7] E. Kablov, O. Ospennikova, V. Piskorskii, D. Korolev, A. Dmitriev, E. Kunitsyna, R. Morgunov. Eur. Phys. J. Plus 131, 40 (2016).

[8] R.B. Morgunov, E.I. Kunitsyna, V.V. Kucheryaev, V.P. Piskorskii, O.G. Ospennikova, E.N. Kablov. Eur. Phys. J. Plus 131, 344 (2016).

[9] Е.Н. Каблов, О.Г. Оспенникова, В.П. Пискорский, И.И. Резчикова, Р.А. Валеев, Е.А. Давыдова. Авиационные материалы и технологии $\mathbf{S 2}, 5$ (2015).

[10] R. Morgunov, A. Talantsev, E. Kunitsyna, V. Piskorskii, O. Ospennikova, E. Kablov. IEEE Transact. Magn. 52, 1 (2016),

[11] B.C. Dodrill. Magn. Busin. \& Technology Mag. (2015).

[12] M.F. de Campos. Mater. Sci. Forum 660-661, 284 (2010).

[13] R. Bodenberger, A. Hubert. Phys. Status Solidi K7-K11, B 44 (1977).

Редактор Т.Н. Василевская 